BNL- -46671

DE92 001773

Modification of the U-Line of the RHIC Injection Line

Jianming $\mathrm{Xu}$

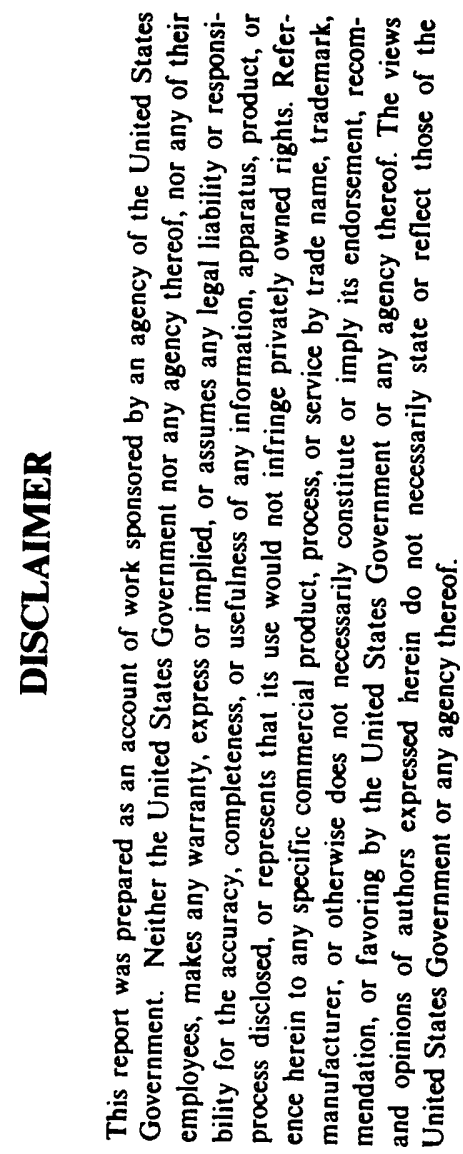

September 1991

R H I C P R O J E C T

Brookhaven National Laboratory

Associated Universities, Inc.

Upton, NY 11973 


\title{
MODIFICATION OF THE.U-LINE OF THE RHIC INJECTION LINE
}

\author{
Jianming $\mathrm{Xu}$ \\ Accelerator Development Department \\ Brookhaven National Laboratory
}

September 1991

The parameters of the U-line of the RHIC injection line with low $\beta$ waist are described in Reference 1. In that lattice, the location of SA is not dispersion free and 14 quadrupoles are needed. This line has been modified to move SA to a dispersion free region (after the 8-degree bend), the length and maximum gradient of quadrupoles have been adjusted to fit the existing quadrupole parameters and the number of quadrupoles is reduced to 12 .

\section{The Effect of Dispersion Functions at SA}

According to Reference 2, the energy loss of $10.4 \mathrm{GeV} / \mathrm{u} \mathrm{Au}$ ion after passing $\mathrm{Au}$ foil $\left(\sim 40 \mathrm{mg} / \mathrm{cm}^{2}\right)$ is $0.65 \mathrm{MeV} / \mathrm{u}$ and the additional $\mathrm{rms}$ energy spread is $0.065 \mathrm{MeV} / \mathrm{u}$. In the lattice of reference 1 , at SA $\eta_{x}=3.816 \mathrm{~m}, \eta_{x}^{\prime}=-0.019, \eta_{y}=0, \eta_{y}^{\prime}=0$. After passing foil SA, the additional rms $\Delta x$ and $\Delta x^{\prime}$ due to additional energy spread are

$$
\begin{aligned}
& \Delta x_{r m s}=0.02 \mathrm{~mm} \\
& \Delta x_{r m s}^{\prime}=1.2 \times 10^{-4} \mathrm{mrad}
\end{aligned}
$$

The induced $\mathrm{rms}$ beam emittance growth is about $6.25 \times 10^{-5} \mathrm{~mm} \cdot \mathrm{mrad}$. The injection beam $\mathrm{rms}$ emittance is $0.13 \mathrm{~mm} \cdot \mathrm{mrad}$. So, the relative emittance growth is only $4.8 \times 10^{-4}$ which is negligible small.

After passing foil SA, the change of the closed orbit due to energy loss are

$$
\begin{aligned}
& \Delta x_{c o}=0.2 \mathrm{~mm} \\
& \Delta x_{c o}^{\prime}=1.2 \times 10^{-3} \mathrm{mrad} .
\end{aligned}
$$

This change of closed orbit after the foil SA should be corrected by proper dipole correctors otherwise this closed orbit shift will introduce a shift of beam center. In order to avoid this problem in the modified U-line, foil SA is moved to a dispersion free region. 


\section{The Parameters of the Modified U-Line}

The parameters of the modified U-line are shown in Table 1, which is the output of MAD program. The $\beta$ value at SA is 6 meters and the maximum $\beta$ value in quadrupoles is 208 meters which is larger than 117 meters in the lattice of reference 1. But considering that, the aperture of the quadrupoles in the $U$-line is $100 \mathrm{~mm}$, the maximum half beam height is $25.8 \mathrm{~mm}$ even when taking $3.2 \mathrm{~mm} \cdot \mathrm{mrad}$ as the beam emittance. This maximum beam height is less than $60 \%$ of the quadrupole aperture and the actual beam emittance is only $0.8 \mathrm{~mm} \cdot \mathrm{mrad}$. So, there is enough safety margin.

The maximum beam cross section is smaller if $\beta$ at SA is larger. The corresponding maximum $\beta$ values for different $\beta$ values at SA are shown in Table 2. The maximum beam cross section may be reduced further if we add some extra quadrupole to strengthen the focusing status if this is necessary.

Table 2:

\begin{tabular}{|cccccc|}
\hline$\beta$ at SA & 6.0 & 6.1 & 6.2 & 6.4 & 6.6 \\
$\beta_{x, \max }$ & 195.6 & 199 & 192.2 & 191.5 & 189.9 \\
$\beta_{y, \max }$ & 207.9 & 199 & 202.2 & 194.4 & 189.3 \\
\hline
\end{tabular}

The parameters of the existing quadrupoles for U-line are shown in Table 3. The parameters of the quadrupoles of this modified lattice are all fit to these existing quadrupoles.

Table 3:

\begin{tabular}{|cc|}
\hline Length $(\mathrm{m})$ & Maximum Gradient (T/M) \\
\hline 0.72 & 15.5 \\
0.74 & 17.5 \\
1.0 & 17.5 \\
\hline
\end{tabular}

\section{References}

1. The Effect of the Stripping Foil SA on the Injection Beam of RHIC, Jianming Xu, AD/RHIC/RD-18, 1990.

2. The Heavy Ion Stripping Foil Requirements between AGS and RHIC, M.J. Rhoades- Brown, AD/RHIC-68, 1990. 


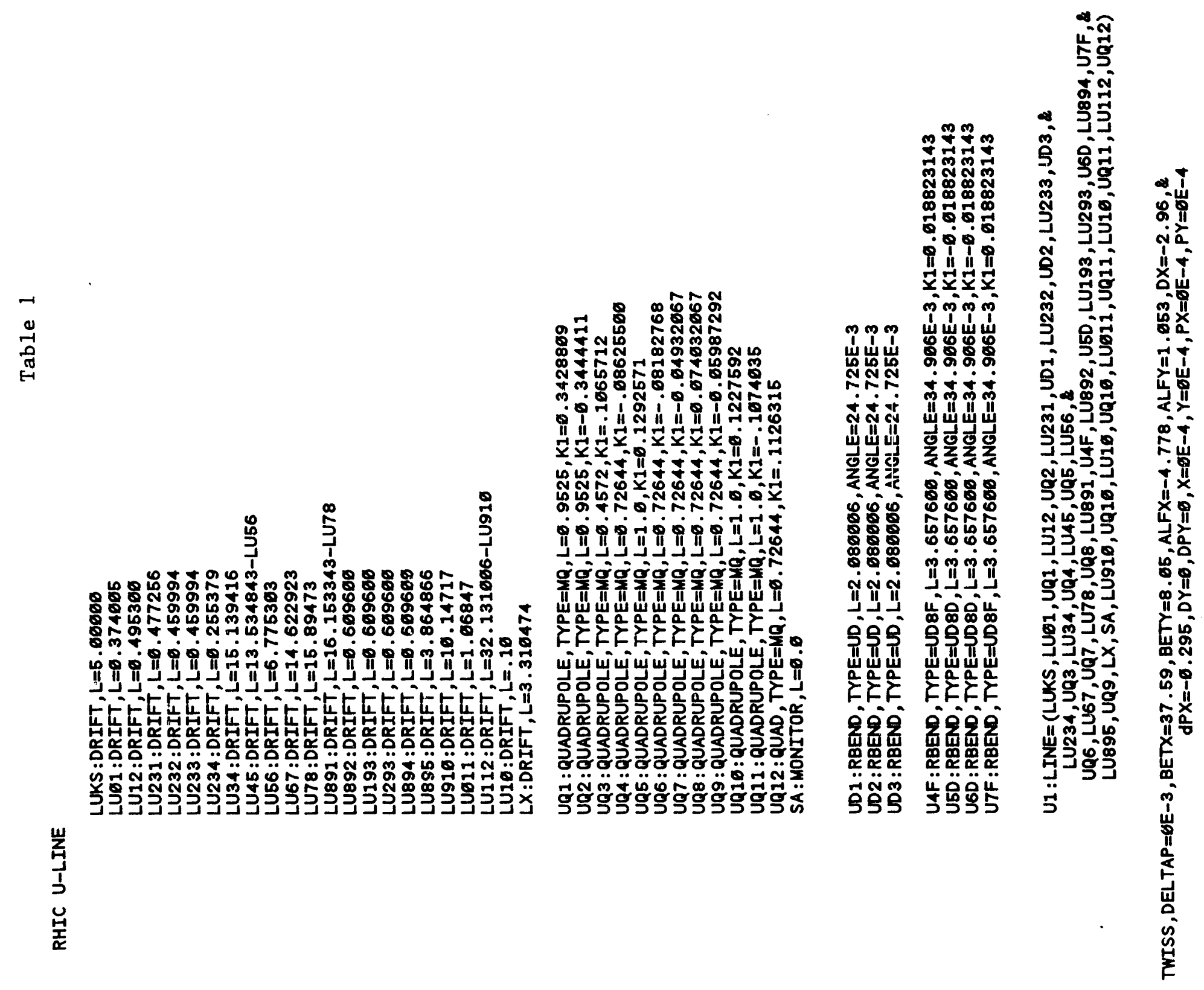




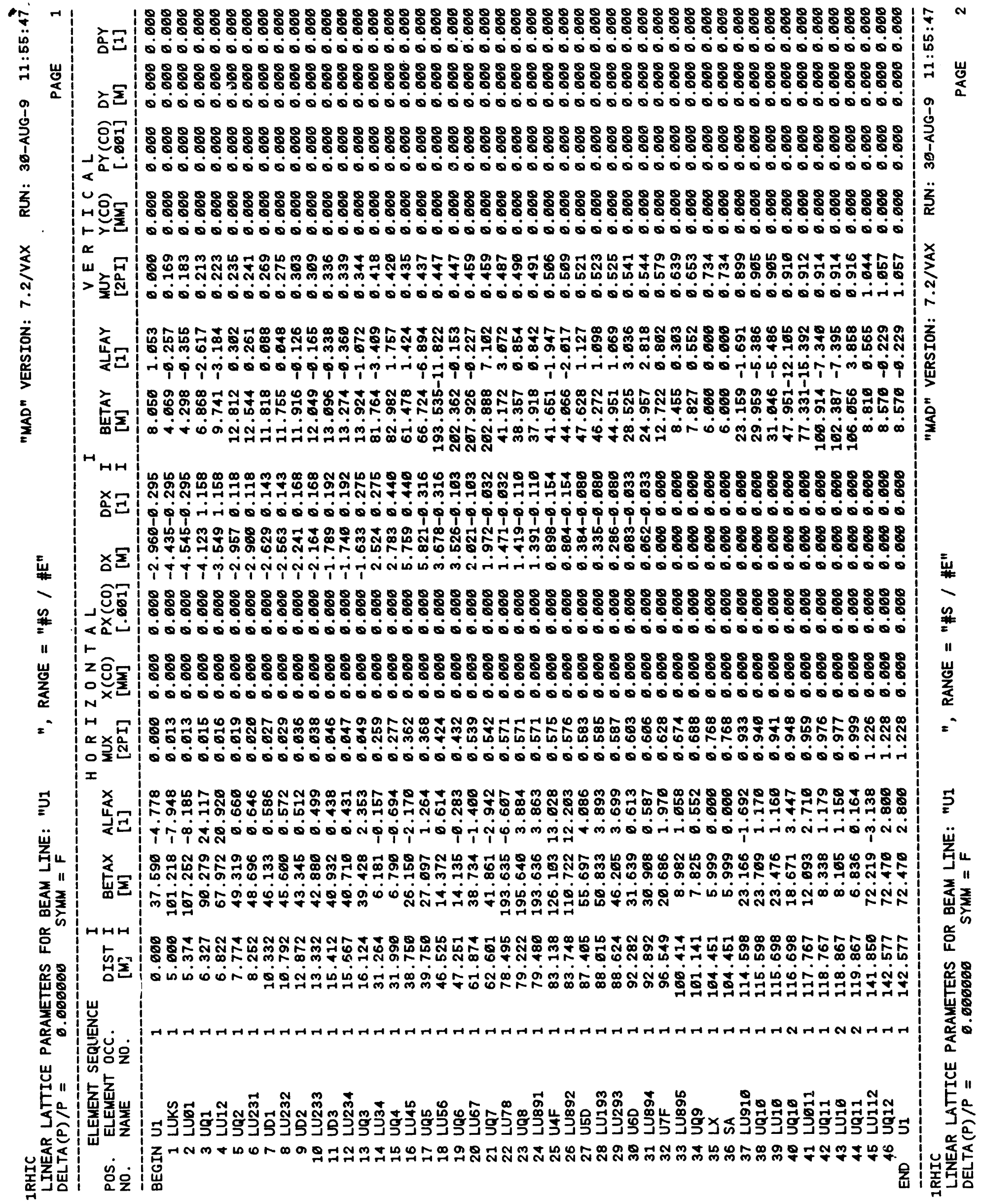




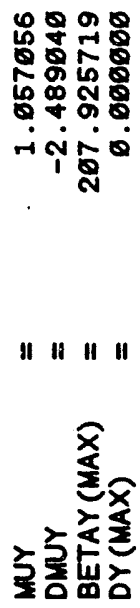

ษัธ์

ลูสูู

$7 \mathrm{~T}$

X

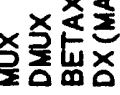

E

ㅇํㅇ

옹요

的昌

พู่

" "

I

学

近

占㟔 

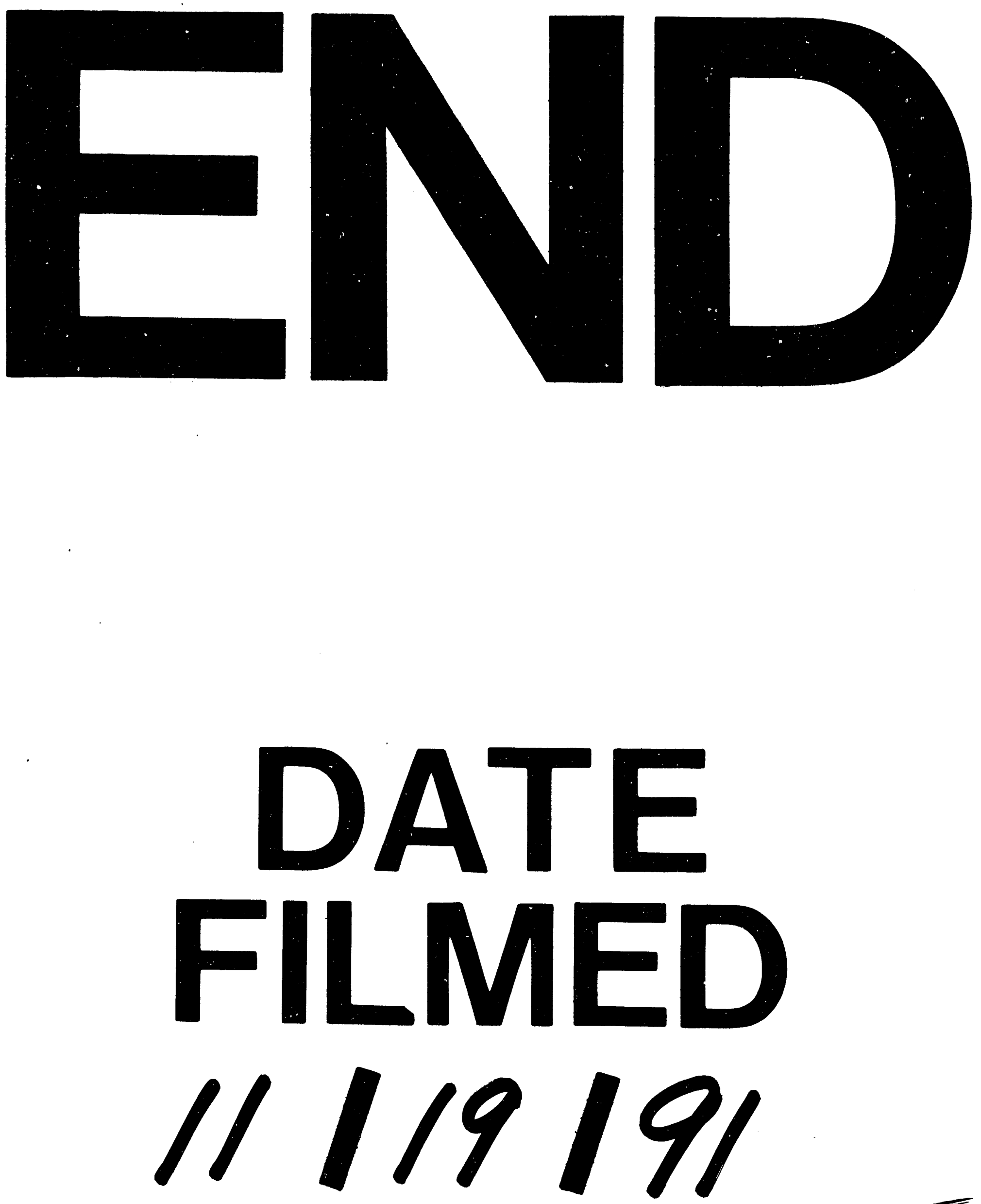
